Nimmt man an, daß die Konvektionsströme für alle Versuche von ähnlicher Größenordnung waren, so fällt ihr störender Einfluß um so stärker ins Gewicht, je geringer der Stofftransport durch Diffusion ist; dies ist besonders bei den tieferen Arbeits- temperaturen der Fall. Allerdings zeigen die aus dem Dampfraum entnommenen Proben, daß eine Trennung innerhalb der Gasphase tatsächlich stattfindet.

\title{
Kinetische Gleidhungen und Integrabilitätsbedingung im Vielzeitenformalismus
}

\author{
D. Frank *, D. Pfirsch und S. Priess \\ Max-Planck-Institut für Physik und Astrophysik, München \\ (Z. Naturforschg. 20 a, 147-150 [1965]; eingegangen am 2. November 1964)
}

\begin{abstract}
An Hand einer Klasse gewöhnlicher Differentialgleichungen und eingehender an Hand eines speziellen Beispieles wird das Vielzeitenverfahren als eine Lösungsmethode der B B G K Y - Gleichungen diskutiert. Insbesondere werden ein von $S_{A N D R I}$ und ein von $S_{u}$ vorgeschlagener Ansatz für die Berechnung von Korrekturen zur LandaU-Gleichung auf ihren Anwendungsbereich hin untersucht.
\end{abstract}

Die BBGKY-Gleichungen eines einatomigen Gases lauten im Falle schwacher Wechselwirkung, d. h. für kleine mittlere potentielle Energie im Vergleich zur mittleren Energie der ungeordneten Bewegung (ihr Verhältnis wird mit $\varepsilon$ bezeichnet, $\varepsilon \ll 1$ ) und für eine Reichweite der Kräfte, die nicht wesentlich größer als der mittlere Teilchenabstand ist

$$
\frac{\partial F_{s}}{\partial t}+K_{s} F_{s}=\varepsilon I_{s} F_{s}+\varepsilon L_{s} F_{s+1} ;
$$

dabei ist $F_{s}=F_{s}\left(\boldsymbol{x}_{1}, \ldots, \boldsymbol{x}_{s} ; \boldsymbol{v}_{1}, \ldots, \boldsymbol{v}_{s} ; t ; \varepsilon\right)$ die $s$-Teilchen-Verteilungsfunktion und $K_{s}, I_{s}, L_{s}$ sind die folgenden Operatoren:

$$
\begin{aligned}
& K_{s}=\sum_{i=1}^{s} \boldsymbol{v}_{i} \cdot \frac{\partial}{\partial \boldsymbol{x}_{i}} \\
& I_{s}=\sum_{1 \leq i<j \leq s} I_{i j} ; \quad I_{i j}=\frac{\partial U\left(\boldsymbol{x}_{i i}\right)}{\partial \boldsymbol{x}_{i}} \cdot\left(\frac{\partial}{\partial \boldsymbol{v}_{i}}-\frac{\partial}{\partial \boldsymbol{v}_{j}}\right) ; \\
& L_{s}=\sum_{i=1}^{s} L_{i, s+1} ; \\
& L_{i, s+1}=\int \mathrm{d} x_{s+1} \mathrm{~d} v_{s+1} \frac{\partial U\left(\boldsymbol{x}_{i, s+1}\right)}{\partial \boldsymbol{x}_{i}} \cdot \frac{\partial}{\partial \boldsymbol{v}_{i}} ; \\
& \boldsymbol{x}_{i j}=\boldsymbol{x}_{i}-\boldsymbol{x}_{j} .
\end{aligned}
$$

* Unser Freund D. F RANK ist kurz vor Abschluß dieser Arbeit am 23.2. 1964 gestorben.

1 N. N. Bogolsubow, Studies in Statistical Mechanics, Vol. 1, Edited by J. Deboer and G. E. Uhlenbeck, North Holland Publishing Co., Amsterdam 1962.

2 E. A. Frieman, Princeton University, Proj. Matt. Rep. Matt. $-106,1961$.
$U$ ist das Wechselwirkungspotential zweier Teilchen mit dem maximalen Wert 1. Der Ort ist in Einheiten der Reichweite der Kräfte, die Geschwindigkeit in Einheiten der mittleren ungeordneten Geschwindigkeit und die Zeit in Einheiten der mittleren Dauer der Wechselwirkung zweier Teilchen gemessen. In der vorliegenden Arbeit soll eine Lösungsmethode für diese Gleichungen untersucht werden, die auf die grundlegenden Überlegungen BogolJuBow's ${ }^{1} \mathrm{zu}$ rückgeht, und die durch Frieman, Sandri und Mitarbeiter $^{2-5}$ auf eine mathematisch durchsichtigere Form gebracht wurde: Die Verteilungsfunktionen $F_{s}$ werden in geeigneter Weise als Funktionen $F_{s}$ $\left(x_{1}, \ldots ; v_{1}, \ldots ; t, \varepsilon t, \varepsilon^{2} t, \ldots\right)$ dargestellt. Auf diese Weise kann man mit $\tau_{v}=\varepsilon^{v} t$ neue Zeitvariable $\tau_{v}$ einführen. Indem man diese $\tau_{v}$ dann als voneinander unabhängig betrachtet, werden die Funktionen $F_{s}$ zu Funktionen über dem ganzen $\tau_{v}$-Raum fortgesetzt. Auf die so erweiterten Funktionen $F_{s}$ wird Störungsrechnung nach $\varepsilon$ angewandt :

$$
\begin{aligned}
F_{s} & =F_{s}{ }^{0}+\varepsilon F_{s}{ }^{1}+\varepsilon^{2} F_{s}{ }^{2}+\ldots, \\
F_{s}{ }^{v} & =F_{s}{ }^{v}\left(\boldsymbol{x}_{1}, \ldots ; \boldsymbol{v}_{1}, \ldots ; \tau_{0}, \tau_{1}, \ldots\right) .
\end{aligned}
$$

3 E. A. Frieman, J. Math. Phys. 4, 411 [1963].

${ }^{4}$ G. SANDRI, The Foundations of Nonequilibrium Statistical Mechanics, Lecture Notes, Rutgers University 1961-1962.

5 C. H. Su, Kinetic Theory of Weakly Coupled Gases, Thesis in the Department of Aeronautical Engineering, Princeton University 1964. 
Man erhält auf diese Weise aus der BBGKY-Hierarchie folgendes Gleichungssystem bis zur dritten Ordnung:

$$
\begin{gathered}
\frac{\partial F_{s}{ }^{0}}{\partial \tau_{0}}+K_{s} F_{s}{ }^{0}=0, \\
\frac{\partial F_{s}{ }^{0}}{\partial \tau_{1}}+\frac{\partial F_{s}{ }^{1}}{\partial \tau_{0}}+K_{s} F_{s}{ }^{1}=I_{s} F_{s}{ }^{0}+L_{s} F_{s+1}^{0}, \\
\frac{\partial F_{s}{ }^{0}}{\partial \tau_{2}}+\frac{\partial F_{s}{ }^{1}}{\partial \tau_{1}}+\frac{\partial F_{s}{ }^{2}}{\partial \tau_{0}}+K_{s} F_{s}{ }^{2}=I_{s} F_{s}{ }^{1}+L_{s} F_{s+1}^{1}, \\
\frac{\partial F_{s}{ }^{0}}{\partial \tau_{3}}+\frac{\partial F_{s}{ }^{1}}{\partial \tau_{2}}+\frac{\partial F_{s}{ }^{2}}{\partial \tau_{1}}+\frac{\partial F_{s}{ }^{3}}{\partial \tau_{0}}+K_{s} F_{s}{ }^{3}=I_{s} F_{s}{ }^{2}+L_{s} F_{s+1}^{2} .
\end{gathered}
$$

Insbesondere ergibt sich für die 1-Teilchenfunktion im Falle, daß $F_{s}{ }^{0}=\prod_{r=1}^{s} F_{1}{ }^{0}(v)$ zur Zeit $\tau_{0}=0$ gilt:

$$
\begin{aligned}
\frac{\partial F_{1}{ }^{0}}{\partial \tau_{0}} & =0, \\
\frac{\partial F_{1}{ }^{0}}{\partial \tau_{1}}+\frac{\partial F_{1}{ }^{1}}{\partial \tau_{0}} & =0, \\
\frac{\partial F_{1}{ }^{0}}{\partial \tau_{2}}+\frac{\partial F_{1}{ }^{1}}{\partial \tau_{1}}+\frac{\partial F_{1}{ }^{2}}{\partial \tau_{0}} & =L_{1} F_{2}{ }^{1}, \\
\frac{\partial F_{1}{ }^{0}}{\partial \tau_{3}}+\frac{\partial F_{1}{ }^{1}}{\partial \tau_{2}}+\frac{\partial F_{1}{ }^{2}}{\partial \tau_{1}}+\frac{\partial F_{1}{ }^{3}}{\partial \tau_{0}} & =L_{1} F_{2}{ }^{2} .
\end{aligned}
$$

Es wird nach Lösungen gefragt, bei denen keine „säkularen Terme“ vorhanden sind. „Säkulare Terme“ sind formal durch Auftreten von positiven Potenzen in den $\tau_{v}$ erklärt.

Wie man an den Gleichungen sieht, bekommt man für ein und dieselbe Funktion Gleichungen mit Ableitungen nach verschiedenen Variablen $\tau_{v}$. Es besteht daher die Frage, ob die Verträglichkeit dieser Differentialgleichungen gleichzeitig mit der Forderung nach Säkularitätenfreiheit zu erfüllen ist. Das Problem ergibt sich hier, sobald man über die zweite Ordnung hinausgeht, d. h. sobald man Korrekturen zur sogenannten LANDAU-Gleichung ${ }^{1,4}$ angeben will.

Um Einblick in den mathematischen Formalismus $\mathrm{zu}$ bekommen, wird im folgenden in Analogie zu den Hierarchiegleichungen eine Klasse gewöhnlicher Differentialgleichungen erster Ordnung diskutiert.

\section{Der Vielzeitenformalismus für eine Klasse gewöhnlicher Differentialgleichungen erster Ordnung}

\section{a) Allgemeiner Formalismus}

Die betrachtete Klasse von Differentialgleichungen wird durch

$$
\mathrm{d} x / \mathrm{d} t=g(x, \varepsilon)
$$

beschrieben, wobei weitere Forderungen an $g$ noch folgen. Entsprechend der Störungsrechnung im Vielzeitenformalismus hat man für $x$ die Entwicklung

$$
x=\sum_{v=0}^{\infty} \varepsilon^{v} x^{v} \text { mit } x^{v}=x^{v}\left(\tau_{0}, \tau_{1}, \ldots\right) .
$$

Die Funktionen $g\left(\sum \varepsilon^{v} x^{v}, \varepsilon\right)$ sollen bezüglich $\varepsilon$ in eine TAYLOR-Reihe entwickelbar sein:

$$
\begin{aligned}
g(x, \varepsilon) & =g\left(x^{0}, 0\right)+\varepsilon\left(g_{\varepsilon}+x^{1} g_{x}\right) \\
& +\frac{\varepsilon^{2}}{2}\left(g_{\varepsilon \varepsilon}+2 x^{1} g_{x \varepsilon}+\left(x^{1}\right)^{2} g_{x x}+2 x^{2} g_{x}\right) \\
& +\frac{\varepsilon^{3}}{6}\left(g_{\varepsilon \varepsilon \varepsilon}+3 x^{1} g_{x \varepsilon \varepsilon}+3\left(x^{1}\right)^{2} g_{x x \varepsilon}+6 x^{2} g_{x \varepsilon}\right. \\
& \left.+\left(x^{1}\right)^{3} g_{x x x}+6 x^{1} x^{2} g_{x x}+6 x^{3} g_{x}\right)+\varepsilon^{4} \ldots .
\end{aligned}
$$

Zur Abkürzung bezeichneten wir $\left.g_{\varepsilon}\right|_{\varepsilon=0},\left.g_{x}\right|_{\varepsilon=0}, \ldots$ mit $g_{\varepsilon}, g_{x}, \ldots$. Um Gleichungen zu erhalten, die ein ähnliches Aussehen haben wie die Hierarchiegleichungen (7) für die 1-Teilchenfunktion, wird gefordert:

$$
\frac{\partial x^{0}}{\partial \tau_{0}}=0, \quad \frac{\partial x^{0}}{\partial \tau_{1}}+\frac{\partial x^{1}}{\partial \tau_{0}}=0 .
$$

Wie man an der $\varepsilon$-Entwicklung von $g$ erkennt, bedeutet das:

$$
g\left(x^{0}, 0\right)=0 \quad \text { und } \quad g_{\varepsilon}\left(x^{0}, 0\right)=0 .
$$

Daraus folgt:

$$
\begin{aligned}
& \left.\frac{\partial n}{\partial x^{n}} g(x, \varepsilon)\right|_{\varepsilon=0}=0, \\
& \frac{\partial n}{\partial x^{n}} g_{\varepsilon}(x, \varepsilon)_{\varepsilon=0}=0, \quad n=0,1,2, \ldots .
\end{aligned}
$$

Wir betrachten also Funktionen $g$ von der Form: $g(x, \varepsilon)=\frac{\varepsilon^{2}}{2} g_{\varepsilon \varepsilon}+\frac{\varepsilon^{3}}{6}\left(g_{\varepsilon \varepsilon \varepsilon}+3 x^{1} g_{x \varepsilon \varepsilon}\right)+\varepsilon^{4} \ldots$.

Bis zur dritten Ordnung in $\varepsilon$ erhält man im Vielzeitenformalismus folgende Gleichungen:

$$
\begin{aligned}
\frac{\partial x^{0}}{\partial \tau_{0}} & =0, \\
\frac{\partial x^{0}}{\partial \tau_{1}}+\frac{\partial x^{1}}{\partial \tau_{0}} & =0, \\
\frac{\partial x^{0}}{\partial \tau_{2}}+\frac{\partial x^{1}}{\partial \tau_{1}}+\frac{\partial x^{2}}{\partial \tau_{0}} & =\frac{1}{2} g_{\varepsilon \varepsilon}, \\
\frac{\partial x^{0}}{\partial \tau_{3}}+\frac{\partial x^{1}}{\partial \tau_{2}}+\frac{\partial x^{2}}{\partial \tau_{1}}+\frac{\partial x^{3}}{\partial \tau_{0}} & =\frac{1}{6} g_{\varepsilon \varepsilon \varepsilon}+\frac{1}{2} x^{1} g_{x \varepsilon \varepsilon} .
\end{aligned}
$$

Dieses System von partiellen Differentialgleichungen wird gelöst für die Anfangsbedingung, daß zur Zeit $t=0$, d. h. für alle $\tau_{v}=0$, alle Funktionen $x^{\nu}$, 
$v \geqq 0$, bekannt sind, und zwar unter der Bedingung, daß im Sinne der Definition nach Gl. (7) keine säkularen Terme auftreten:

Aus (14) folgt, daß $x^{0}$ unabhängig von $\tau_{0}$ ist, also

$$
x^{0}=x^{0}\left(\tau_{1}, \tau_{2}, \ldots\right),
$$

damit ergibt sich aus (15) weiter

$x^{0}=x^{0}\left(\tau_{2}, \tau_{3}, \ldots\right), \quad x^{1}=x^{1}\left(\tau_{1}, \tau_{2}, \ldots\right)$

und aus (16) schließlich

$$
\begin{aligned}
& x^{2}=x^{2}\left(\tau_{1}, \tau_{2}, \ldots\right), \\
& \frac{\partial x^{0}}{\partial \tau_{2}}+\frac{\partial x^{1}}{\partial \tau_{1}}=\frac{1}{2} g_{\varepsilon \varepsilon},
\end{aligned}
$$

woraus man nach Integration über $\tau_{1}$ erhält

$$
x^{1}=x^{1}\left(\tau_{2}, \tau_{3}, \ldots\right), \quad \frac{\partial x^{0}}{\partial \tau_{2}}=\frac{1}{2} g_{\varepsilon \varepsilon} .
$$

(24) entspricht gerade der normalen kinetischen Gleichung bei der Lösung der Hierarchiegleichungen. Aus (17) folgt jetzt weiter

$$
\begin{gathered}
x^{3}=x^{3}\left(\tau_{1}, \tau_{2}, \ldots\right), \\
\frac{\partial x^{0}}{\partial \tau_{3}}+\frac{\partial x^{1}}{\partial \tau_{2}}+\frac{\partial x^{2}}{\partial \tau_{1}}=\frac{1}{6} g_{\varepsilon \varepsilon \varepsilon}+\frac{1}{2} x^{1} g_{x \varepsilon \varepsilon},
\end{gathered}
$$

und somit nach Integration über $\tau_{1}$

$$
\begin{gathered}
x^{2}=x^{2}\left(\tau_{2}, \tau_{3}, \ldots\right), \\
\frac{\partial x^{0}}{\partial \tau_{3}}+\frac{\partial x^{1}}{\partial \tau_{2}}=\frac{1}{6} g_{\varepsilon \varepsilon \varepsilon}+\frac{1}{2} x^{1} g_{x \varepsilon \varepsilon} .
\end{gathered}
$$

Aus der Vertauschbarkeit der Reihenfolge der Ableitungen von $x^{0}$ nach $\tau_{2}$ und $\tau_{3}$ erhält man für $x^{1}$ als Funktion von $\tau_{2}$ eine Differentialgleichung zweiter Ordnung. Es ist nämlich

$\frac{\partial^{2} x^{0}}{\partial \tau_{3} \partial \tau_{2}}=\frac{1}{2} \boldsymbol{g}_{\varepsilon \varepsilon x} \frac{\partial x^{0}}{\partial \tau_{3}}=\frac{1}{2} g_{\varepsilon \varepsilon x}\left(\frac{1}{6} g_{\varepsilon \varepsilon \varepsilon}+\frac{1}{2} x^{1} g_{x \varepsilon \varepsilon}-\frac{\partial x^{1}}{\partial \tau_{2}}\right)$

und

$$
\begin{aligned}
\frac{\partial^{2} x^{0}}{\partial \tau_{2} \partial \tau_{3}}=-\frac{\partial^{2} x^{1}}{\partial \tau_{2}^{2}}+\frac{1}{6} g_{\varepsilon \varepsilon \varepsilon x} \frac{1}{2} g_{\varepsilon \varepsilon} & +\frac{1}{2} \frac{\partial x^{1}}{\partial \tau_{2}} g_{x \varepsilon \varepsilon} \\
& +\frac{1}{4} x^{1} g_{x x \varepsilon \varepsilon} g_{\varepsilon \varepsilon}
\end{aligned}
$$

und damit

$$
\begin{aligned}
\frac{\partial^{2} x^{1}}{\partial \tau_{2}{ }^{2}}-g_{\varepsilon \varepsilon x} \frac{\partial x^{1}}{\partial \tau_{2}} & +\frac{1}{4}\left(g_{\varepsilon \varepsilon x}^{2}-g_{\varepsilon \varepsilon} g_{x x \varepsilon \varepsilon}\right) x^{1} \\
& +\frac{1}{12}\left(g_{\varepsilon \varepsilon x} g_{\varepsilon \varepsilon}-g_{\varepsilon \varepsilon} g_{\varepsilon \varepsilon \varepsilon x}\right)=0 .
\end{aligned}
$$

6 Dieses Problem war von den Verfassern in einem Vortrag auf der „VI. Conférence Internationale sur les Phénomènes d'Ionisation dans les Gaz", Orsay/Frankreich, aufgegriffen worden, und zwar direkt im Rahmen der kinetischen Gleichungen bei schwacher Wechselwirkung. Dort wurde bewie-
Aus der Differentialgleichung zweiter Ordnung ist unter Berücksichtigung der Anfangsbedingung $x^{1}$ als Funktion von $\tau_{2}$ so zu bestimmen, daß keine Säkularitäten auftreten. Dann erhält man aus (28) eine Differentialgleichung für $x^{0}$ in seiner Abhängigkeit von $\tau_{3} . \mathrm{SU}^{5}$ nimmt an

$$
\partial x_{0} / \partial \tau_{3}=0 \text {. }
$$

Hierbei ist natürlich die Integrabilitätsbedingung bezüglich $\tau_{2}$ und $\tau_{3}$ erfüllt. Man bestimmt $x^{\mathbf{1}}\left(\tau_{2}\right)$ aus der aus (28) folgenden Differentialgleichung

$$
\partial x^{1} / \partial \tau_{2}=\frac{1}{6} g_{\varepsilon \varepsilon \varepsilon}+\frac{1}{2} x^{1} g_{x \varepsilon \varepsilon} .
$$

Damit $x^{1}\left(\tau_{2}\right)$ frei von Säkularitäten ist, muß in diesem Fall gefordert werden:

$$
\lim _{\tau_{2} \rightarrow \infty} g_{x \varepsilon \varepsilon}<0 .
$$

$\mathrm{S}_{\text {ANDRI }}{ }^{4}$ macht die Annahme

$$
x_{1} \equiv 0 .
$$

Da $x^{1} \equiv 0$ dann also eine Lösung der Dgl. (20) ist, muß gelten

folglich

$$
g_{\varepsilon \varepsilon x} g_{\varepsilon \varepsilon}-g_{\varepsilon \varepsilon} g_{\varepsilon \varepsilon \varepsilon x}=0,
$$

$$
g_{\varepsilon \varepsilon}=c g_{\varepsilon \varepsilon \varepsilon} \quad \text { mit } \quad c=\text { const . }
$$

Das ist eine stark einschränkende Bedingung an die Klasse der Funktionen $g$, da $g_{\varepsilon \varepsilon}$ und $g_{\varepsilon \varepsilon \varepsilon}$ als Funktionen in $x$ voneinander völlig unabhängig sind ${ }^{6}$.

\section{b) Ein Beispiel}

An Hand eines speziellen Beispieles sollen die von uns angegebene, die Susche und die Sandrische Methode noch einmal diskutiert werden. Es sei $g(x, \varepsilon)$ der folgende Ausdruck:

$$
g(x, \varepsilon)=-\frac{1}{6} \varepsilon^{2}(x-1)(3+\varepsilon(1+x)) .
$$

Das ursprüngliche Problem (8) hat dann die exakte Lösung

$$
x=\frac{1+(3+\varepsilon) k \exp \left\{-\left(\frac{1}{2} \varepsilon^{2}+\frac{1}{3} \varepsilon^{3}\right) t\right\}}{1-k \varepsilon \exp \left\{-\left(\frac{1}{2} \varepsilon^{2}+\frac{1}{3} \varepsilon^{3}\right) t\right\}},
$$

wobei $k$ durch die Anfangsbedingung bestimmt ist:

$$
x=\frac{1+k(3+\varepsilon)}{1-k \mathrm{e}} \text { für } t=0 .
$$

sen, daß die SANDrischen Gleichungen für $\partial f^{0} / \partial \tau_{2}$ und $\partial f_{0} / \partial \tau_{3}$ nicht miteinander verträglich sind. Versehentlich wurden jedoch fehlerhafte Ausgangsgleichungen benutzt, so daß jener Beweis hinfällig ist. 
Nach Anwendung des Vielzeitenformalismus und der Störungsrechnung erhält man an Stelle der Gln. (24) und (31) :

$$
\partial x^{0} / \partial \tau_{2}=\frac{1}{2}\left(1-x^{0}\right)
$$

und $\frac{\partial^{2} x^{1}}{\partial \tau_{2}{ }^{2}}+\frac{\partial x^{1}}{\partial \tau_{2}}+\frac{1}{4} x^{1}-\frac{1}{12}\left(x^{0}-1\right)^{2}=0$.

Aus (41) folgt für $x^{0}$ in seiner Abhängigkeit von $\tau_{2}$

$$
x^{0}=c e^{-\tau_{2} / 2}+1, \quad c=c\left(\tau_{3}, \tau_{4}, \ldots\right),
$$

und damit als allgemeine Lösung von (42)

$$
x^{1}=\left(a+b \tau_{2}\right) e^{-\tau_{2} / 2}+\frac{c^{2}}{3} e^{-\tau_{2}},
$$

$a, b$ sind Funktionen von $\tau_{3}, \tau_{4}, \ldots$.

Da keine „Säkularitäten“ auftreten sollen, muß $b=0$ sein. Wir betrachten also nur Lösungen von der Form

$$
x^{1}=a e^{-\tau_{2} / 2}+\frac{c^{2}}{3} e^{-\tau_{2}} .
$$

So bekommen wir nach (28) als Differentialgleichung für $x^{0}$ als Funktion von $\tau_{3}$

$$
\partial x^{0} / \partial \tau_{3}=-\frac{1}{3} c e^{-\tau_{2} / 2},
$$

woraus sich über (43) eine Differentialgleichung für $c\left(\tau_{3}\right)$ ergibt:

Also ist

$$
\partial c / \partial \tau_{3}=-\frac{1}{3} c .
$$

$$
c=c_{0} e^{-\tau_{3} / 3}, \quad c_{0}=c_{0}\left(\tau_{4}, \tau_{5}, \ldots\right) .
$$

Die Funktionen $c_{0}$ und $a$ werden aus der Anfangsbedingung (40) bestimmt, es folgt

$$
c_{0}=3 k, \quad a=2 k .
$$

Damit erhalten wir als Approximation der Lösung (39)

$$
\begin{aligned}
x^{0}+\varepsilon x^{1}=(1 & \left.+3 k e^{-\tau_{2} / 2-\tau_{3} / 3}\right) \\
& +\varepsilon\left(2 k e^{-\tau_{2} / 2}+3 k^{2} e^{-\tau_{2}-2 \tau_{3} / 3}\right) .
\end{aligned}
$$

Diese Approximation entspricht der folgenden Entwicklung der exakten Lösung $x$ :

$$
\begin{aligned}
x=\left(1+(3+\varepsilon) k e^{-\left(\tau_{2} / 2+\tau_{3} / 3\right)}\right) & \cdot\left(1+k \varepsilon e^{-\left(\tau_{2} / 2+\tau_{3} / 3\right)}+\ldots\right),
\end{aligned}
$$

d. h. der Ausdruck für $x$ ist wie $1 /(1-a)=1+a+\ldots$ mit $a=k \varepsilon e^{-\left(\tau_{2} / 2+\tau_{3} / 3\right)}$ entwickelt.

Nach der Methode von Su erhält man als approximierende Lösung von (39) $x^{0}=\gamma e^{-\tau_{2} / 2}+1, \quad \gamma=\gamma\left(\tau_{4}, \ldots\right)$ wegen $\partial x^{0} / \partial \tau_{3}=0$,

und

$$
\begin{array}{r}
x^{1}=e^{-\tau_{2} / 2}\left(\alpha-\frac{1}{3} \gamma^{2}\right)+\gamma^{2} e^{-\tau_{2}}-\frac{1}{3} \gamma \tau_{2} e^{-\tau_{2} / 2}, \\
\alpha=\alpha\left(\tau_{3}, \tau_{4}, \ldots\right) .
\end{array}
$$

Aus der Anfangsbedingung (40) folgt

Damit ist

$$
\gamma=3 k, \quad \alpha=2 k+3 k^{2} .
$$

$$
x^{0}=3 k e^{-\tau_{2} / 2}+1
$$

und

$$
x^{1}=2 k e^{-\tau_{2} / 2}+3 k^{2} e^{-\tau_{2}}-k \tau_{2} e^{-\tau_{2} / 2} .
$$

In der formalen Handhabung der Säkularitätenmethode wäre der letzte Ausdruck schon nicht mehr zulässig.

Die Approximation $x^{0}+\varepsilon x^{1}$ entspricht in diesem Fall folgender Entwicklung der exakten Lösung (39) :

$$
\begin{array}{r}
x=\left(1+(3+\varepsilon) k e^{-\tau_{2} / 2}\left(1-\frac{1}{3} \varepsilon \tau_{2}+\ldots\right)\right) \\
\cdot\left(1+k \varepsilon e^{-\tau_{2} / 2}(1+\ldots)+\ldots\right) .
\end{array}
$$

Diese Entwicklung kann man aus der zu dem von uns angegebenen, allgemeineren Verfahren erhalten, wenn man in jener $e^{-\varepsilon \tau_{2} / 3}$ für $e^{-\tau_{3} / 3}$ schreibt und diese letzte Funktion wieder entwickelt. Die Lösung von $\mathrm{Su}$ führt gerade wegen des formal säkularen Termes für größere Werte von $\tau_{3}$ zu einer schlechteren Approximation der exakten Lösung als das allgemeine Verfahren, während für kleinere Werte von $\tau_{3}$ die Abweichungen noch unerheblich sind. Interessiert man sich also für nicht zu große Werte von $\tau_{3}$, so ist die Susche Methode anwendbar und wegen ihrer vergleichsweise großen Einfachheit der allgemeinen Methode vorzuziehen.

Die SANDRIsche Methode ist für das hier betrachtete Beispiel nicht anwendbar, da die Bedingung (37) nicht erfüllt ist. Man erhielte nach SANDRI:

woraus folgt

$$
\partial x^{0} / \partial \tau_{2}=\frac{1}{2}\left(1-x^{0}\right)
$$

$$
x^{0}=c e^{-\tau_{2} / 2}+1, \quad c=c\left(\tau_{3}, \tau_{4}, \ldots\right),
$$

und $\quad \partial x^{0} / \partial \tau_{3}=\frac{1}{6}\left(1-\left(x^{0}\right)^{2}\right)$,

damit $\quad \partial c / \partial \tau_{3}=-\frac{1}{6} c^{2} e^{-\tau_{2} / 2}-\frac{1}{3} c$.

Diese Art der $\tau_{2}$-Abhängigkeit von $c$ kann innerhalb der $\tau_{3}$-Skala aber nicht mehr vernachlässigt werden. 


\section{NOTIZEN}

\section{Aufladung kleiner Teilchen im Elektronen- mikroskop}

Ludwig Reimer

Physikalisches Institut der Universität Münster (Westf.)

(Z. Naturforschg. 20 a, 151-152 [1965]; eingeg. am 27. November 1964)

$\mathrm{M}_{\mathrm{AHL}}$ und $\mathrm{W}_{\mathrm{EITSCH}}{ }^{1}$ haben mit einer elektronenoptischen Schattenmethode gezeigt, daß isolierende Schichten (z. B. Formvar, Kollodium oder $\mathrm{SiO}$ ) im Elektronenstrahl Aufladungen erfahren, die sich örtlich und zeitlich verändern („fluktuierende Ladungen“). Leitende Kohleschichten zeigen diese Erscheinung nicht. Bei der elektronenoptischen Schattenbeobachtung von $\mathrm{MgO}$ - und NaCl-Teilchen auf Kohle-Trägerfolien haben wir jedoch einen stationäre Aufladung der Teilchen beobachtet, über die in der vorliegenden Mitteilung berichtet werden soll.

Mit dem Doppelkondensor des S i e me n s-Elmiskop I wurde ein engster Strahlquerschnitt als Projektionszentrum etwa $200 \mathrm{~mm}$ oberhalb des Objektes erzeugt. Die Abbildung erfolgte nur mit der Zwischenlinse. Mit dieser konnten Ebenen bis $50 \mathrm{~mm}$ oberhalb und unterhalb des Objektes abgebildet werden. Mit wachsender Defokussierung unterhalb des Objektes wird das Bild einer Netzmasche größer. Das Bild der Teilchen steigt jedoch nicht im gleichen Maße an. Es kann sogar der Fall eintreten, daß die durch die Aufladung der Teilchen hervorgerufene Ablenkung so groß ist, daß sich die Strahlen hinter dem Teilchen in einigen $\mathrm{cm}$ Entfernung überschneiden und zum „Verschwinden" des Projektionsbildes führen. Bei würfelförmigen Teilchen ruft die inhomogene Verteilung des elektrischen Feldes Veränderungen des Projektionsbildes hervor, wie sie in Abb. 1 * in einer Defokussierungsserie zu erkennen sind. Die Aufnahmen sind so nachvergrößert, daß die Gesamtvergrößerung - erkenntlich am Abstand der beiden Teilchen - konstant ist. Man erkennt eine Konzentration der Intensität längs der Würfeldiagonalen (Abb. $1 \mathrm{c}-1 \mathrm{e}$ ) und eine Verkleinerung des Schattenbildes senkrecht zu den Würfelkanten. In Abb. 1 a ist eine Ebene oberhalb des Objektes abgebildet. Durch die rückwärtig verlängerten Strahlengänge entsteht ein scheinbar vergrößertes Bild der Teilchen. Man beachte auch den hellen Saum um das Objekt.

Um die Ursache dieser Aufladung zu klären, wurde versucht, diese quantitativ zu erfassen. Nimmt man an, daß die Oberfläche des Teilchens auf konstantem Potential $V$ gegenüber der leitenden Kohleschicht liegt, so kann man das den Würfel umgebende elektrische Feld $E$ mit der Methode des elektrolytischen Troges

1 H. MAhL u. W. Weitsch, Naturwiss. 47, 301 [1960]; Z. Naturforschg. 15 a, 1051 [1960].

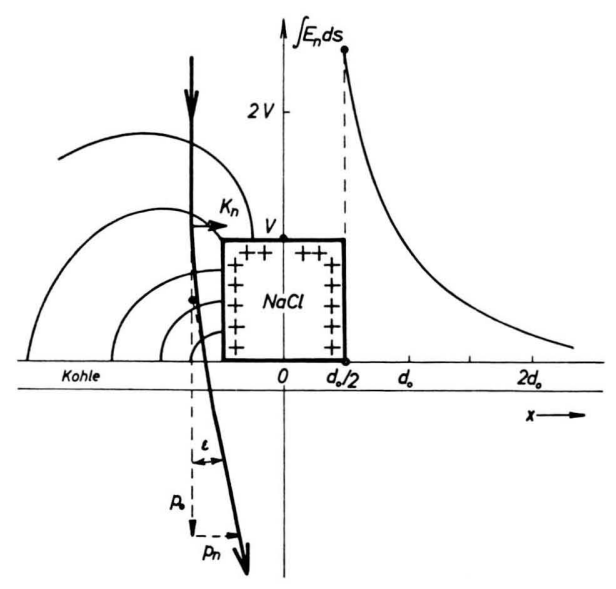

Abb. 2. Skizze zur Ablenkung des Elektrons im inhomogenen elektrischen Feld um ein NaCl-Teilchen (links) und Verteilung von $\int E_{\mathrm{n}} \mathrm{d} s$ als Funktion des Abstandes $x$ vom Teilchen (rechts)

experimentell ermitteln. Die zum Elektronenstrahl senkrechte Komponente $E_{\mathrm{n}}$ wirkt auf das Elektron mit der Kraft $K_{\mathrm{n}}=e E_{\mathrm{n}}$ (Abb. 2, links). Während des Fluges durch das inhomogene Feld wird auf das Elektron ein Impuls

$$
p_{\mathrm{n}}=\int K_{\mathrm{n}} \mathrm{d} t=e \int E_{\mathrm{n}} \mathrm{d} t=\frac{e}{v_{0}} \int E_{\mathrm{n}} \mathrm{d} s
$$

übertragen $\left(v_{0}=\sqrt{2 e U_{0} / m}=\right.$ Bahngeschwindigkeit des Elektrons, $U_{0}=$ Beschleunigungsspannung). Mit dem Impuls $p_{0}=m v_{0}$ in Strahlrichtung ergibt sich eine $\mathrm{Ab}$ lenkung des Elektronenstrahles um den Winkel

$$
\varepsilon=\frac{p_{n}}{p_{0}}=\frac{\int E_{\mathrm{n}} \mathrm{d} s}{2 U_{0}} .
$$

Die Messung im elektrolytischen Trog wurde so durchgeführt, daß $\int E_{\mathrm{n}} \mathrm{d} s$ durch graphische Integration erhalten werden konnte. Abb. 2 rechts zeigt den Verlauf dieser Größe in Abhängigkeit vom Abstand $x$ von dem würfelförmigen Teilchen. Aus der Porssonschen Differentialgleichung $\Delta U=0$ ersieht man, da $ß$ am Rand eines Teilchens $\int E_{\mathrm{n}} \mathrm{d} s$ nur von der Aufladung $V$ der Teilchenoberfläche abhängt, aber unabhängig von der Teilchengröße ist.

Mit $a=$ Abstand Objekt - Leuchtschirm, $b=$ Objekt Zwischenlinse und $c=$ Projektionszentrum-Objekt kann man geometrisch eine Beziehung zwischen dem Bilddurchmesser $d_{1}$ des Teilchens und dem Abstand $s$ der von der Zwischenlinse abgebildeten Ebene vom

* Abb. 1 auf Tafel S. 152 a. 
Objekt angeben $\left(D_{1}=\right.$ Bild einer Netzmasche, $D_{0}$, $d_{0}=$ wahre Abmessungen von Netzmasche bzw. Teilchen) :

$$
\frac{d_{1}}{D_{1}}=\frac{d_{0}}{D_{0}}-\frac{2 \varepsilon}{D_{0}} \cdot \frac{s}{1+s / c} .
$$

In Abb. 3 ist $d_{1} / D_{1}$ gegen $s /(1+s / c)$ aufgetragen. Es ergaben sich für NaCl-Teilchen verschiedener Kantenlänge die erwarteten Geraden, aus deren Steilheit der Ablenkwinkel $\varepsilon$ und mit (2) $\int E_{\mathrm{n}} \mathrm{d} s$ zu entnehmen ist $\left(U_{0}=100 \mathrm{kV}\right)$. Die in Abb. 3 verzeichneten Werte lie-

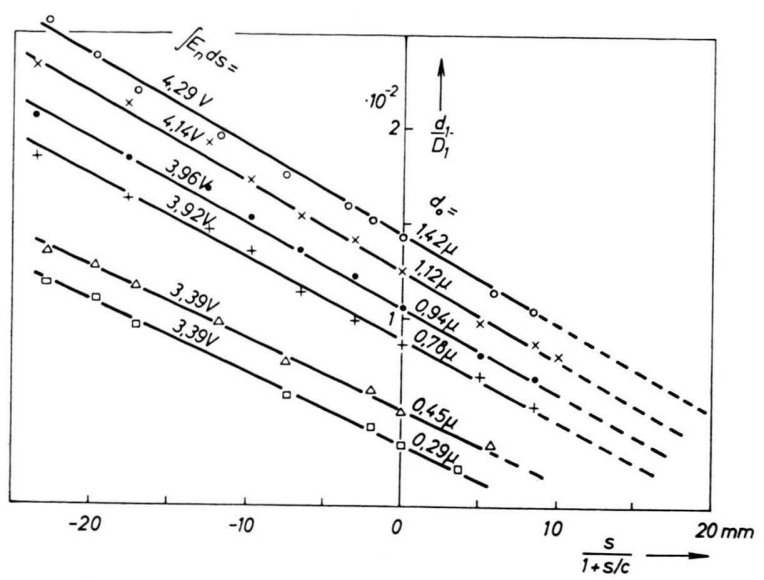

Abb. 3. Messungen des Bilddurchmessers $d_{1}$ der Teilchen in Abhängigkeit von der Defokussierung $s$ (positives $s$ unterhalb des Objektes), $U_{0}=100 \mathrm{kV}$.

gen um 4 Volt. Nach Abb. 2 ist $\int E_{\mathrm{n}} \mathrm{d} s$ am Rand eines Teilchens etwa 2,5-mal größer als die Aufladung in Volt. Man kann daher auf eine positive Aufladung der $\mathrm{NaCl}$-Teilchen von ca. 1,6 V gegenüber der Kohlefolie schließen. Diese läßt sich mit Sekundärelektronenemission erklären. Nach VöLKER ${ }^{2}$ haben bei der Bestrahlung von Metallschichten mit Elektronen im Energiebereich 8 bis $40 \mathrm{keV}$ die Sekundärelektronen eine Energieverteilung mit einem Maximum bei $1-2 \mathrm{eV}$, aber ca. $50 \%$ der Elektronen treten noch mit einer Energie größer als $5 \mathrm{eV}$ aus. Da durch den Elektronenbeschuß sicher eine schwache Leitfähigkeit in den NaClKristallen induziert wird, wird die positive Aufladung sich in einem dynamischen Gleichgewicht ausbilden.

Ein Überdampfen der Teilchen mit einer leitenden Kohleschicht unterdrückt den Aufladungseffekt. Kontaminationsschichten, welche während der Bestrahlung aus den organischen Restgasen niedergeschlagen werden, haben keinen erkennbaren Einfluß. Sie zeigen

2 M. VöLker, Dissertation, Techn. Universität, Berlin 1959.

3 S. F. Kern u. R. A. Kern, J. Appl. Phys. 21, 705 [1950].

4 G. Müller u. K. Meyerhoff, Nature, Lond. 201, 590 [1964]. bekanntlich eine sehr geringe elektrische Leitfähigkeit. Außer bei $\mathrm{NaCl}, \mathrm{MgO}$ u. a. Alkalihalogeniden wurde diese Erscheinung noch an kugelförmigen PVC-Teilchen beobachtet. Polystyrol-Latex zeigte dagegen keine Aufladung. Auch Platinpulver-Teilchen lassen eine schwache, allerdings negative Aufladung erkennen. Diese ist als Kontaktpotential zwischen der Kohleschicht und dem Platinteilchen mit einer höheren Austrittsarbeit zu deuten. Wegen der unregelmäßigen Gestalt der Pulverteilchen ist eine quantitative Bestimmung nicht möglich. Nach den Literaturwerten ist dagegen die Austrittsarbeit von $\mathrm{NaCl}$ und Kohle in der gleichen Größenordnung. Daher wird man zur Deutung der Erscheinungen an diesen Teilchen die oben diskutierte Sekundärelektronenemission heranziehen müssen.

Bei normaler elektronenmikroskopischer Beobachtung mit eingeschaltetem Objektiv beträgt bei einer Aufladung von $2 \mathrm{~V}$ die scheinbare Verkleinerung des Teilchenbildes nur einige $\AA$. Um erkennbare Verkleinerungen im elektronenmikroskopischen Bild zu erhalten - für Latexteilchen sind derartige Möglichkeiten von $\mathrm{KERN}_{\mathrm{ER}}$ und $\mathrm{K}_{\mathrm{ERN}}{ }^{3}$ diskutiert -, sind also wesentlich größere Aufladungen erforderlich. Obwohl die Potentialdifferenz zwischen Teilchen und Kohleschicht nur ca. $2 \mathrm{~V}$ beträgt, herrscht an der Teilchenoberfläche natürlich eine hohe elektrische Feldstärke. Für kugelförmige Teilchen mit $1 \mu$ Durchmesser läßt sie sich nach der Beziehung $E=U / r$ zu $4 \cdot 10^{4} \mathrm{~V} / \mathrm{cm}$ abschätzen.

Neben dieser Möglichkeit zur Erfassung sehr kleiner Aufladungen elektronenmikroskopischer Objekte interessiert diese Wirkung der Teilchen als „Mikrolinse" auch im Zusammenhang mit einer von MüLler und Meyerhoff ${ }^{4}$ diskutierten anomalen Kontrasterscheinung bei der Negativ-Kontrastierung. Nur müßte es sich hierbei um Aufladungen handeln, die mehr als 100-mal stärker sind, da bei normaler Fokussierung beobachtet wird. Es läßt sich schwer ein Mechanismus für derartig hohe Aufladungen angeben, da oberhalb Feldstärken von $10^{5} \mathrm{~V} / \mathrm{cm}$ elektrische Durchschläge zu erwarten sind. Durch eine Arbeit von Lippert ${ }^{5}$ wurde die obige Deutung des anomalen Kontrastes auch in Frage gestellt.

Jönsson und HoffmanN ${ }^{6}$ beobachten ebenfalls eine positive Aufladung bei isolierenden Oberflächenschichten auf leitender Unterlage. Am Rand eines Folienloches konnten Umlenkwinkel von $2,4 \cdot 10^{-6}$ gemessen werden. Bei unseren Versuchen beträgt $\varepsilon=2 \cdot 10^{-5}$. Der Unterschied in der Größenordnung ist auf geometrische Unterschiede zurückzuführen, die sich auf den Wert von $\int E_{\mathrm{n}} \mathrm{d} s$ auswirken.

5 W. Lippert, Naturwiss. 51, 408 [1964].

${ }^{6}$ C. Jönsson u. H. Hoffmann, Optik 21, 432 [1964]. 


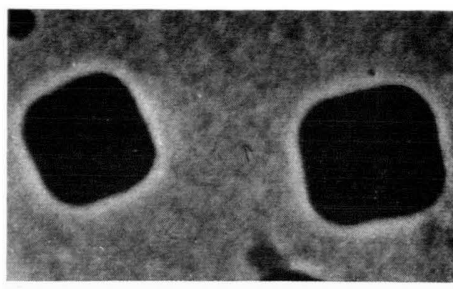

a)

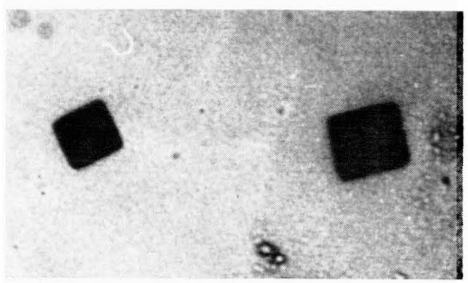

b)

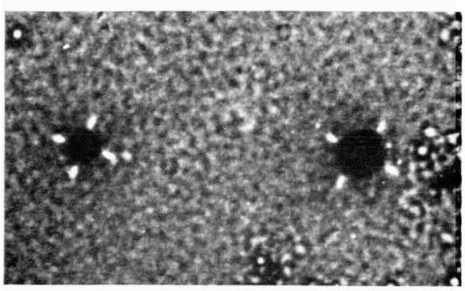

c)

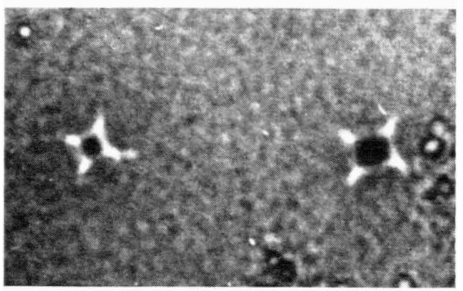

d)

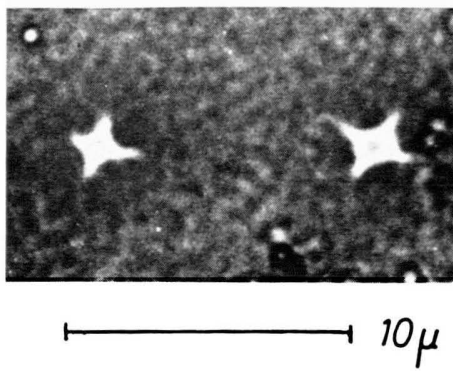

e)

Abb. 1. Defokussierungserie mit der Zwischenlinse von 2 würfelförmigen NaCl-Teilchen, a) $50 \mathrm{~mm}$ oberhalb des Objektes, b) fokussiert, c) $20 \mathrm{~mm}$, d) $30 \mathrm{~mm}$, e) $50 \mathrm{~mm}$ unterhalb des Objektes $\left(U_{0}=80 \mathrm{kV}\right)$. 
K. Müller, Orientierte Sauerstoffadsorption an Silber-Aufdampfschichten (S. 153).

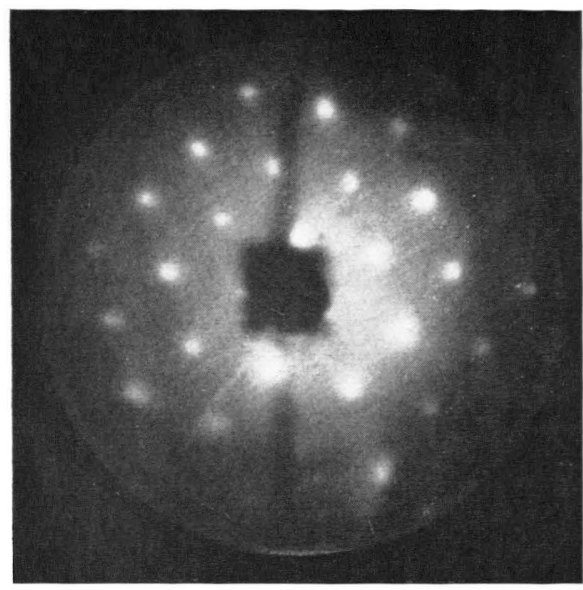

Abb. 1 a.

Glimmer (001)

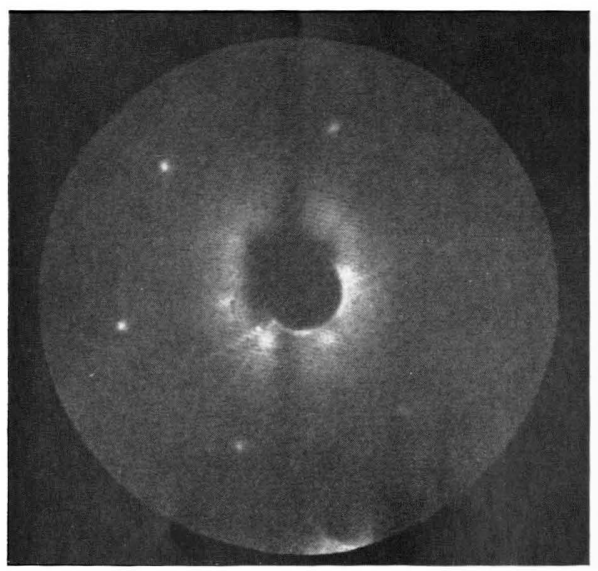

Abb. 2 a.

Silber (111) gasbelegt, Struktur I

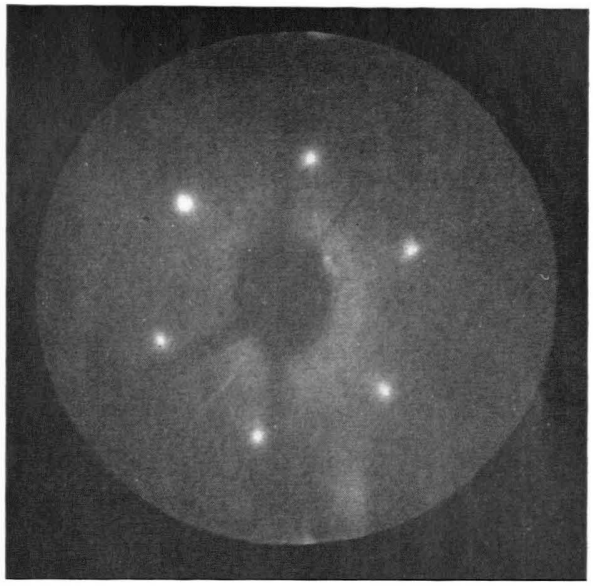

Abb. 1 b.

Beugung bei $110 \mathrm{eV}$.

Silber (111)

saubere Oberfläche

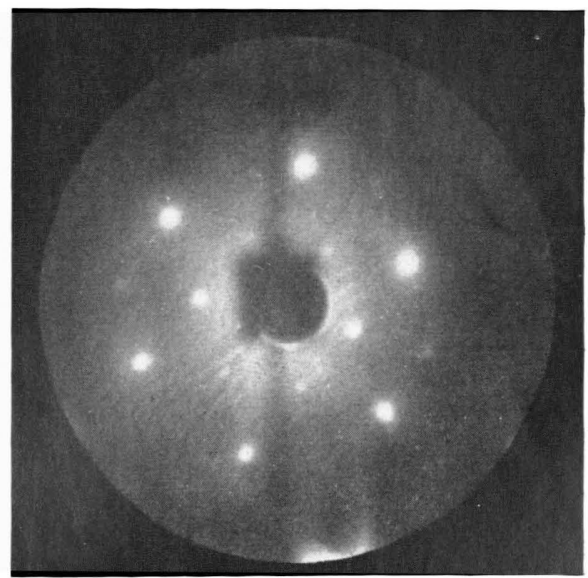

Abb. 2 b.

Silber (111)

gasbelegt, Struktur II 